\title{
Neuropsychiatric symptoms are early indicators of an upcoming metabolic decline in Alzheimer's disease
}

\author{
Kok Pin Ng ${ }^{1,2,3}$, Tharick A. Pascoal ${ }^{1,2}$, Sulantha Mathotaarachchi ${ }^{1,2}$, Yiong Huak Chan ${ }^{4}$, Lai Jiang ${ }^{5,6}$, \\ Joseph Therriault1,2, Andrea L. Benedet 1,2, Monica Shin ${ }^{1,2}$, Nagaendran Kandiah³, Celia M. T. Greenwood ${ }^{5,6}$, \\ Pedro Rosa-Neto ${ }^{1,2}$, Serge Gauthier ${ }^{1,2^{*}}$ and Dominantly Inherited Alzheimer Network
}

\begin{abstract}
Background: Neuropsychiatric symptoms (NPS) are increasingly recognized as early non-cognitive manifestations in the Alzheimer's disease (AD) continuum. However, the role of NPS as an early marker of pathophysiological progression in $A D$ remains unclear. Dominantly inherited $A D(D I A D)$ mutation carriers are young individuals who are destined to develop AD in future due to the full penetrance of the genetic mutation. Hence, the study of DIAD mutation carriers enables the evaluation of the associations between pure AD pathophysiology and metabolic correlates of NPS without the confounding effects of co-existing pathologies. In this longitudinal study, we aimed to identify regional brain metabolic dysfunctions associated with NPS in cognitively intact DIAD mutation carriers.

Methods: We stratified 221 cognitively intact participants from the Dominantly Inherited Alzheimer's Network according to their mutation carrier status. The interactions of NPS measured by the Neuropsychiatric InventoryQuestionnaire (NPI-Q), age, and estimated years to symptom onset (EYO) as a function of metabolism measured by $\left[{ }^{18} \mathrm{~F}\right]$ flurodeoxyglucose $\left(\left[{ }^{18} \mathrm{~F}\right] \mathrm{FDG}\right)$ positron emission tomography, were evaluated by the mixed-effects regression model with family-level random effects in DIAD mutation carriers and non-carriers. Exploratory factor analysis was performed to identify the neuropsychiatric subsyndromes in DIAD mutation carriers using the NPI-Q subcomponents. Then the effects of interactions between specific neuropsychiatric subsyndromes and EYO on metabolism were evaluated with the mixed-effects regression model.

Results: A total of 119 mutation carriers and 102 non-carriers were studied. The interaction of higher NPI-Q and shorter EYO was associated with more rapid declines of global and regional $\left[{ }^{18} \mathrm{~F}\right] \mathrm{FDG}$ uptake in the posterior cingulate and ventromedial prefrontal cortices, the bilateral parietal lobes and the right insula in DIAD mutation carriers. The neuropsychiatric subsyndromes of agitation, disinhibition, irritability and depression interacted with the EYO to drive the $\left[{ }^{18} \mathrm{~F}\right] \mathrm{FDG}$ uptake decline in the DIAD mutation carriers. The interaction of NPI and EYO was not associated with $\left[{ }^{18} \mathrm{~F}\right] \mathrm{FDG}$ uptake in DIAD mutation non-carriers.

(Continued on next page)
\end{abstract}

\footnotetext{
* Correspondence: serge.gauthier@mcgill.ca

'Alzheimer's Disease Research Unit, McGill Centre for Studies in Aging, McGill University, Montréal, Québec, Canada

${ }^{2}$ Translational Neuroimaging Laboratory, The McGill University Research Centre for Studies in Aging, Montreal, Canada

Full list of author information is available at the end of the article
}

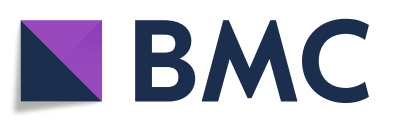

(c) The Author(s). 2021 Open Access This article is licensed under a Creative Commons Attribution 4.0 International License, which permits use, sharing, adaptation, distribution and reproduction in any medium or format, as long as you give appropriate credit to the original author(s) and the source, provide a link to the Creative Commons licence, and indicate if changes were made. The images or other third party material in this article are included in the article's Creative Commons licence, unless indicated otherwise in a credit line to the material. If material is not included in the article's Creative Commons licence and your intended use is not permitted by statutory regulation or exceeds the permitted use, you will need to obtain permission directly from the copyright holder. To view a copy of this licence, visit http://creativecommons.org/licenses/by/4.0/ The Creative Commons Public Domain Dedication waiver (http://creativecommons.org/publicdomain/zero/1.0/) applies to the data made available in this article, unless otherwise stated in a credit line to the data. 
(Continued from previous page)

Conclusions: The NPS in cognitively intact DIAD mutation carriers may be a clinical indicator of subsequent metabolic decline in brain networks vulnerable to AD, which supports the emerging conceptual framework that NPS represent early manifestations of neuronal injury in AD. Further studies using different methodological approaches to identify NPS in preclinical AD are needed to validate our findings.

Keywords: Neuropsychiatric symptoms, Dominantly inherited Alzheimer's disease, Metabolic correlates

\section{Background}

Neuropsychiatric symptoms (NPS) are frequently observed in mild cognitive impairment (MCI) and dementia stages of Alzheimer's disease (AD) [1-4] and are associated with greater functional impairment, poorer quality of life, accelerated cognitive decline [5-7] and a more significant degree of $\mathrm{AD}$ neurodegeneration [4]. In cognitively normal individuals, developing NPS later in life may potentially increase the risk of cognitive decline [8-11]. Therefore, NPS are being increasingly considered as a non-cognitive manifestation in the early stages of $\mathrm{AD}$ when one is cognitively intact [12]. However, the roles of NPS as early clinical manifestations of pathophysiological progression of $\mathrm{AD}$ in cognitively normal individuals remain unclear. Our recent study showed that NPS in preclinical sporadic AD individuals preceded hypometabolism in the posterior cingulate cortex, a key brain region involved in the $\mathrm{AD}$ process [13]. Hence, investigating NPS as an early manifestation of metabolic decline in an independent cognitively intact cohort known to have $\mathrm{AD}$ pathophysiology will further advance the emerging conceptual framework in which NPS constitute an early clinical manifestation of $\mathrm{AD}$.

Dominantly inherited AD (DIAD) is a familial AD due to autosomal dominant mutations in APP, PSEN1 or PSEN2 and cognitively intact individuals who are DIAD mutation carriers are destined to develop $\mathrm{AD}$ in future due to the full penetrance of the genetic mutation [14]. Similar to the late-onset sporadic AD [15], the pathophysiology of DIAD begins to accumulate in the preclinical stage of the disease when the carriers are cognitively normal $[16,17]$. In DIAD, early behavioural changes have been reported in mutation carriers with mild cognitive symptoms, in whom the NPS increase as their disease progresses [18]. Furthermore, the DIAD mutation carriers are younger than individuals with sporadic $\mathrm{AD}$ and are less likely to have other medical conditions such as cerebrovascular diseases [19]. Therefore, by enrolling cognitively normal DIAD mutation carriers, the associations between pure AD pathophysiology and metabolic correlates of NPS in the preclinical stage of $\mathrm{AD}$ can be evaluated without the confounding effects of co-existing pathologies.

In this study, we set out to perform this observation in cognitively normal DIAD mutation carriers and noncarriers from the Dominantly Inherited Alzheimer Network (DIAN) [20].

\section{Methods}

\section{Participants}

Data analyzed in this study were obtained from the DIAN Data Freeze 11. The DIAN observational study is an international multi-site study that enrolls family members who have parents with a mutated gene known to cause DIAD [20]. The study participants may or may not be mutation carriers and they may or may not have cognitive symptoms. The participants underwent standardized clinical and cognitive testing, brain imaging, and biological fluid collection (blood, cerebrospinal fluid [CSF]) to determine the sequence of changes in presymptomatic mutation carriers who are destined to develop AD.

In this study, we selected cognitively normal DIAD mutation carriers and non-carriers from the DIAN cohort with clinical dementia rating (CDR) [21] score of 0 and mini-mental state examination [22] score $\geq 24$ [18, 23]. Individuals who were mildly symptomatic $(\mathrm{CDR}=$ $0.5)$ or overtly symptomatic $(C D R>0.5)$ were excluded. The results of neuropsychiatric inventory-questionnaire (NPI-Q) and $\left[{ }^{18} \mathrm{~F}\right]$ Flurodeoxyglucose (FDG) positron emission tomography (PET) performed at the first visit and at subsequent yearly follow-ups (if available) for each participant were analyzed.

\section{Ethical approval and patient consent}

The DIAN study was approved by the Institutional Review Boards of all of the participating institutions. Informed written consent was obtained from all participants at each site.

\section{Neuropsychiatric assessments}

The NPI-Q is an informant-based assessment tool that measures the presence and severity of behavioural disturbances in 12 behavioural domains of agitation, anxiety, apathy, appetite changes, delusions, depression, disinhibition, abnormally elevated mood, hallucinations, irritability, repetitive motor behaviours, and sleep behaviour changes in clinical settings, within the past month [24]. Higher NPI-Q scores represent greater severity of NPS.

\section{Estimated years to symptom onset (EYO) of DIAN}

The estimated age of onset of cognitive impairment in the cognitively normal individuals from the DIAN was 
calculated based on the mean mutation age of symptom onset and/or the parental age of symptom onset according to the following steps as described previously [25]:

(i) At any study visit, the EYO was calculated as the age at visit subtracting the mean mutation age of symptom onset if the individual's mutation is known, which is available in the DIAN database. (ii) If the individual's mutation is not available in the DIAN database (e.g. the mutation has not been previously reported or other member age of onset not available), then at any study visit, the EYO was calculated as the age at visit subtracting the parental age of symptom onset. The shorter the EYO, the closer the proximity of the individual's time of clinical disease.

\section{Genetic analysis}

Sequencing of the APP, PSEN1 and PSEN2 genes was performed by the DIAN Genetics Core investigators as previously described [18], to reveal the presence of disease-causing mutation in individuals at the risk of AD.

\section{CSF analysis}

CSF levels of $A \beta_{1-42}$, total tau (t-tau) and p-tau181 were measured by immunoassay by the DIAN Biomarker Core at the Washington University, using the Luminex bead-based multiplexed xMAP technology (INNO-BIA AlzBio ${ }^{\text {Tw }}$, Innogenetics, Ghent, Belgium) as previously described [17].

\section{MRI and PET methods}

MRI and PET standard acquisition protocols have been described in the DIAN website. T1-weighted MRI images corrected for field distortions were processed with the CIVET image processing pipeline [26] and the PET images were processed with an established image processing pipeline described previously [27]. The preprocessed images from the DIAN database were spatially normalized to the Montreal Neurological Institute (MNI) 152 standardized space by using the transformations obtained for PET native to MRI native space and the MRI native to the MNI 152 space. The $\left[{ }^{18} \mathrm{~F}\right] \mathrm{FDG}$ PET standardized uptake value ratio (SUVR) maps were then generated using the pons as the reference region $[28,29]$. The global brain glucose uptake was calculated by averaging the $\left[{ }^{18} \mathrm{~F}\right]$ FDG SUVR within several brain regions characteristic to the $\mathrm{AD}$ process, including the precuneus, pre-frontal, orbitofrontal, parietal, temporal, anterior, and posterior cingulate cortices.

\section{Statistical analysis}

The descriptive statistics and frequency distributions of baseline demographics, mutation characteristics and CSF $\mathrm{AD}$ biomarkers were summarized and compared between DIAD mutation carriers and non-carriers using family-level random-effect models for both continuous and categorical measurements using the STATA 15.0 software. Principal components were derived for the variables NPI-Q and EYO to resolve collinear relationships.

The linear mixed effect models with family-level random effects evaluated the interactions between NPI-Q (total and sub-scale scores individually and as a whole), age and EYO on FDG SUVR in the mutation carriers and non-carriers. We modelled FDG SUVR as a function of the interactions of NPI-Q, age and EYO and covariates, where FDG SUVR $i j$ denotes the FDG uptake for the $j$ th person from the $i$ th family, NPI- $\mathrm{Q}_{i j}$ indicates the severity of NPS, age $e_{i j}$ indicates the age of participant at the time of study visit, $\mathrm{EYO}_{i j}$ indicates the years to estimated age of symptom onset and $\mathrm{X}_{i j}$ represents fixed effect covariates for gender, education, $A P O E \varepsilon 4$ status and family mutation type (APP, PSEN1 and PSEN2):

$$
\begin{aligned}
\mathrm{FDG} \mathrm{SUVR}_{i j} \sim & \beta_{0}+\beta_{1}\left(\mathrm{NPI}-\mathrm{Q}_{i j}\right)+\beta_{2}\left(\mathrm{EYO}_{i j}\right) \\
& +\beta_{3}\left(\mathrm{Age}_{i j}\right) \\
& +\beta_{4}\left(\mathrm{NPI}-\mathrm{Q}_{i j} \times \mathrm{EYO}_{i j}\right) \\
& +\beta_{5}\left(\mathrm{NPI}-\mathrm{Q}_{i j} \times \mathrm{Age}_{i j}\right) \\
& +\beta_{6}\left(\mathrm{EYO}_{i j} \times \mathrm{Age}_{i j}\right) \\
& +\beta_{7}\left(\mathrm{NPI}-\mathrm{Q}_{i j} \times \mathrm{EYO}_{i j} \times \mathrm{Age}_{i j}\right) \\
& +\beta_{8}\left(\mathrm{X}_{i j}\right)+F_{i}+\varepsilon_{i j}
\end{aligned}
$$

where $F_{i}$ represents a random effect for all individuals from family $i$, and $\varepsilon_{i j}$ is the residual error assumed to be independent and normally distributed for all individuals.

The family-level random effect accounts for the correlations between individuals within the same family. Although correlations between family members might vary with the relationship type, due to the fairly small sizes of the families, this was modelled with a single random effect.

Voxel-based statistical analyses were then performed using the R Statistical Software Package version 3.3.0 with the RMINC library [30], to test the interactions of NPI-Q, age and EYO on FDG SUVR in the DIAD mutation carriers and non-carriers. All voxel-based regression analyses were corrected for multiple comparisons using random field theory [31] at $p<0.001$.

Exploratory factor analysis was performed on the subcomponents of NPI-Q to identify the neuropsychiatric subsyndromes within the DIAD mutation carriers. This exploratory analysis aims to determine the multidimensional relationships of the NPI-Q sub-components and their overall effects over the individual contributions on the outcomes of interest. Linear mixed effect models with family-level random effects were then used to 
evaluate the interactions of specific neuropsychiatric subsyndromes and EYO on FDG SUVR in the mutationcarrier group.

\section{Results}

Baseline demographics, mutation characteristics and CSF

\section{AD biomarkers}

Two hundred and twenty-one cognitively intact individuals (119 [53.85\%] DIAD mutation carriers and 102 [46.15\%] non-carriers) were included in this study. Twenty-nine individuals had year 1, 24 had year 2, 46 had year 3, 7 had year 4, 4 had year 5 and 3 had year 6 followup data. The baseline demographics, APOE \&4 carrier status, and AD CSF biomarker characteristics are summarized in Table 1. The NPI-Q scores among the DIAD mutation carriers and non-carriers are summarized in Table 2. The DIAD mutation carriers and non-carriers did not differ significantly in age, gender, education and $A P O E$ $\varepsilon 4$ status. As expected, the DIAD mutation carriers had lower CSF $A \beta_{1-42}$, as well as higher CSF p-tau181 and CSF $t$-tau levels than the non-carriers. The NPI-Q total and sub-scale scores did not differ significantly between the mutation carriers and non-carriers.

\section{Interactions among NPI-Q, EYO and age on global $\left[{ }^{18} \mathrm{~F}\right] \mathrm{FDG}$ uptake}

The interaction between NPI-Q and EYO was significantly associated with global $\left[{ }^{18} \mathrm{~F}\right]$ FDG SUVR only in the
DIAD mutation carriers. We found that higher NPI-Q and shorter EYO were associated with a more rapid decline of global $\left[{ }^{18} \mathrm{~F}\right] \mathrm{FDG}$ uptake $(\beta=-0.029,95 \% \mathrm{CI}-$ 0.054 to $-0.004, p=0.024$ ) in the DIAD mutation carriers but not in the non-carriers $(\beta=-0.008,95 \% \mathrm{CI}-$ 0.044 to $0.027, p=0.641$ ) (Table 3 ). The NPI-Q subscales whose interaction with EYO was significantly associated with global $\left[{ }^{18} \mathrm{~F}\right] \mathrm{FDG}$ uptake were anxiety, apathy, depression and irritability for the mutation group. Upon including all the sub-scales in one multivariate model, no significant interactions were observed. We did not find a statistically significant association of interaction between NPI-Q and age with global $\left[{ }^{18} \mathrm{~F}\right]$ FDG uptake in either DIAD mutation carriers or non-carriers. We also did not find a statistically significant association of interaction between NPI-Q, age and EYO with global $\left[{ }^{18} \mathrm{~F}\right]$ FDG uptake in either DIAD mutation carriers or non-carriers.

\section{Associations of NPI-Q and EYO with regional $\left[{ }^{18} \mathrm{~F}\right] \mathrm{FDG}$ uptake}

The voxel-based analysis indicated that the interaction between higher NPI-Q and shorter EYO was associated with regional $\left[{ }^{18} \mathrm{~F}\right] \mathrm{FDG}$ uptake decline in the posterior cingulate cortex (PCC), ventromedial prefrontal cortex (vmPFC), bilateral parietal lobes and right insula in DIAD mutation carriers (Fig. 1). There was no statistically significant association of interaction between NPI-

Table 1 Baseline demographics and sample characteristics of cognitively intact DIAD mutation carriers and non-carriers

\begin{tabular}{|c|c|c|c|}
\hline & DIAD mutation non-carriers $(n=102)$ & DIAD mutation carriers $(n=119)$ & $p$ value \\
\hline Age, years, mean (SD) & $38.99(10.75)$ & $36.45(9.24)$ & 0.060 \\
\hline Male, $n(\%)$ & $44(43.13)$ & $58(48.73)$ & 0.405 \\
\hline Education, years, mean (SD) & $15.15(2.88)$ & $14.92(3.05)$ & 0.566 \\
\hline APOE carrier status & & & 0.600 \\
\hline APOE $\varepsilon 2 / \varepsilon 2, \varepsilon 2 / \varepsilon 3, \varepsilon 3 / \varepsilon 3$ carriers, $n(\%)$ & $72(70.06)$ & $83(69.7)$ & \\
\hline APOE $\varepsilon 2 / \varepsilon 4$ carriers, $n(\%)$ & $6(5.88)$ & $4(3.36)$ & \\
\hline APOE $\varepsilon 3 / \varepsilon 4, \varepsilon 4 / \varepsilon 4$ carriers, $n$ (\%) & $24(23.52)$ & $32(26.89)$ & \\
\hline Parental age of onset, years, mean (SD) & $46.85(6.37)$ & $48.32(7.25)$ & 0.179 \\
\hline EYO, years, mean (SD) & $-9.40(11.92)$ & $-11.51(9.40)$ & 0.078 \\
\hline DIAD mutation type & & & 0.863 \\
\hline$A P P, n(\%)$ & $20(19.60)$ & $20(16.80)$ & \\
\hline PS1, n (\%) & $69(67.65)$ & $83(69.75)$ & \\
\hline PS2, n (\%) & $13(12.75)$ & $16(13.45)$ & \\
\hline MMSE, mean, (SD) & $29.39(0.83)$ & $29.08(1.19)$ & 0.052 \\
\hline $\mathrm{CSF} A \beta_{1-42}$, mean, pg/ml (SD)^ & $461.14(138.27)$ & $363.66(166.72)$ & $<0.001$ \\
\hline CSF p-tau181, mean, pg/ml (SD) ${ }^{+}$ & $29.64(11.96)$ & $53.44(30.65)$ & $<0.001$ \\
\hline CSF t-tau, mean, pg/ml (SD) ${ }^{\dagger}$ & $54.86(25.47)$ & $92.06(62.16)$ & $<0.001$ \\
\hline
\end{tabular}

$P$ values were assessed using family-level random-effects models for the continuous variables and categorical variables, taking into account the analysis of multiple family members within the families. CSF cerebrospinal fluid, EYO estimated years to symptom onset, MMSE mini-mental state examination

${ }^{\wedge} \mathrm{CSF} A \beta_{1-42}$ data for the baseline visit were not available for 19 mutation carriers and 23 non-carriers

${ }^{+}$CSF p-tau181 \& CSF t-tau data for the baseline visit were not available for 18 mutation carriers and 21 non-carriers 
Table 2 Neuropsychiatric symptoms among cognitively intact DIAD mutation carriers and non-carriers

\begin{tabular}{llll}
\hline & DIAD mutation non-carriers $(\boldsymbol{n}=\mathbf{1 0 2})$ & DIAD mutation carriers $(\boldsymbol{n}=\mathbf{1 1 9})$ & $\boldsymbol{p}$ value \\
\hline NPI-Q Score & $0.64(1.23)$ & $0.88(2.19)$ & \\
NPI-Q Sub-scale & & & 0.338 \\
Agitation & $0.07(0.29)$ & $0.08(0.40)$ & 0.699 \\
Anxiety & $0.10(0.35)$ & $0.11(0.33)$ & 0.856 \\
Apathy & $0.01(0.09)$ & $0.04(0.27)$ & 0.295 \\
Appetite & $0.10(0.41)$ & $0.10(0.39)$ & 0.525 \\
Delusion & $0.00(0.00)$ & $0.01(0.09)$ & - \\
Depression & $0.13(0.43)$ & $0.13(0.46)$ & 0.748 \\
Disinhibition & $0.01(0.09)$ & $0.03(0.27)$ & 0.609 \\
Elation & $0.02(0.13)$ & $0.00(0.00)$ & 0.055 \\
Hallucination & $0.00(0.00)$ & $0.00(0.00)$ & - \\
Iritability & $0.12(0.40)$ & $0.18(0.56)$ & 0.207 \\
Motor & $0.02(0.19)$ & $0.05(0.28)$ & 0.366 \\
Sleep changes & $0.07(0.35)$ & $0.14(0.47)$ & 0.195 \\
\hline
\end{tabular}

Mixed Model with family-level random-effects, corrected for age, gender, $A P O E$, education and family mutation type

$N P I-Q$ neuropsychiatric inventory-questionnaire

$\mathrm{Q}$ and EYO, with regional $\left[{ }^{18} \mathrm{~F}\right] \mathrm{FDG}$ uptake in DIAD mutation non-carriers. There was no statistically significant association of interactions between NPI-Q and age, and among NPI-Q, age and EYO, with regional $\left[{ }^{18} \mathrm{~F}\right] \mathrm{FDG}$ uptake, in either DIAD mutation carriers or non-carriers.

\section{Factor analysis of NPI-Q subcomponents}

A four-factor solution was applied to the NPI-Q subcomponents, which explained $70 \%$ of the variance. The neuropsychiatric subsyndromes derived from the factor analysis were (1) agitation, disinhibition, irritability and depression; (2) anxiety, apathy, depression, motor behaviours, and sleep behaviour changes; (3) delusion, sleep behaviour changes and irritability; and (4) appetite and anxiety (Table 4). After including all the subsyndromes in one multivariate model (with family-level as randomeffects, adjusted for gender, APOE, education and family mutation type), only the interaction of the neuropsychiatric subsyndromes agitation, disinhibition, irritability and depression with shorter EYO was associated with the decline of global $\left[{ }^{18} \mathrm{~F}\right] \mathrm{FDG}$ uptake $(\beta=-0.044,95 \%$ CI -0.071 to $-0.017, p=0.001)$ in the DIAD mutation carriers (Table 5).

\section{Discussion}

The present study showed that NPS may be early clinical manifestations of subsequent metabolic dysfunction in brain regions susceptible to AD pathophysiology in cognitively intact DIAD mutation carriers. In these individuals who were destined to develop $\mathrm{AD}$, the more severe the NPS and the shorter the EYO to AD dementia onset, the more rapid the metabolic decline in the PCC, vmPFC, bilateral parietal lobes and right insular. We found that the interaction of the neuropsychiatric subsyndromes agitation, disinhibition, irritability and depression with shorter EYO was associated with a decline of global metabolic uptake over time.

Accumulating evidence has demonstrated the importance of NPS as predictors of cognitive decline in cognitively normal individuals. In the population-based Mayo Clinic Study of Aging, the presence of NPS such as agitation, apathy, anxiety, irritability and depression at baseline increased the risk of incident MCI compared to those without NPS [8]. In the Alzheimer's Disease Cooperative Study Prevention Instrument Project, anxiety and depression at baseline predicted CDR conversion to $\geq 0.5$ in cognitively intact older subjects over a 4-year follow-up [32], while among the National Alzheimer's Coordinating Centers cognitively normal (NACC) volunteers, over 59\% developed NPS before the diagnosis of any cognitive disorder, with depression and irritability being the most common NPS to precede cognitive diagnoses [10]. NPS among cognitively normal individuals may also represent an early manifestation of progressive metabolic dysfunction. In cognitively normal persons aged $>70$ years, depressive and anxiety symptoms are associated with decreased FDG uptake in AD-related regions [33]. In a recent study of preclinical sporadic AD individuals, we found that NPS driven by sleep behavior and irritability domains were associated with metabolic dysfunctions in the limbic network and predicted hypometabolism in the PCC [13]. Therefore, our present findings further support the emerging conceptual framework that NPS are early non-cognitive manifestations of subsequent metabolic decline in AD. 
Table 3 Associations of interactions of NPI-Q and EYO with global $\left[{ }^{18} \mathrm{~F}\right] \mathrm{FDG}$ uptake in cognitively intact DIAD mutation carriers and non-carriers

\begin{tabular}{|c|c|c|c|c|c|c|}
\hline & \multicolumn{3}{|c|}{ DIAD mutation non-carriers } & \multicolumn{3}{|c|}{ DIAD mutation carriers } \\
\hline & Regression estimate & $95 \% \mathrm{Cl}$ & $p$ value & Regression estimate & $95 \% \mathrm{Cl}$ & $p$ value \\
\hline NPI-Q & -0.013 & $-0.040,0.014$ & 0.345 & -0.012 & $-0.030,0.006$ & 0.195 \\
\hline Age & -0.054 & $-0.085,-0.022$ & 0.001 & -0.078 & $-0.108,-0.048$ & $<0.001$ \\
\hline EYO & -0.020 & $-0.050,0.010$ & 0.186 & -0.073 & $-0.105,-0.041$ & $<0.001$ \\
\hline NPI-Q X EYO & -0.008 & $-0.044,0.027$ & 0.641 & -0.029 & $-0.054,-0.004$ & 0.024 \\
\hline \multicolumn{7}{|l|}{ NPI-Q subscales } \\
\hline Agitation & 0.004 & $-0.017,0.025$ & 0.406 & 0.004 & $-0.018,0.027$ & 0.690 \\
\hline Age & -0.057 & $-0.088,-0.026$ & $<0.001$ & -0.077 & $-0.107,-0.046$ & $<0.001$ \\
\hline EYO & -0.020 & $-0.050,0.009$ & 0.170 & -0.076 & $-0.110,-0.043$ & $<0.001$ \\
\hline Agitation X EYO & -0.012 & $-0.028,0.005$ & 0.164 & -0.004 & $-0.020,0.011$ & 0.600 \\
\hline Anxiety & -0.009 & $-0.036,0.017$ & 0.498 & -0.011 & $-0.028,0.006$ & 0.202 \\
\hline Age & -0.055 & $-0.086,-0.024$ & 0.001 & -0.074 & $-0.104,-0.043$ & $<0.001$ \\
\hline EYO & -0.016 & $-0.047,0.014$ & 0.282 & -0.077 & $-0.110,-0.043$ & $<0.001$ \\
\hline Anxiety X EYO & -0.013 & $-0.039,0.013$ & 0.310 & -0.029 & $-0.054,-0.005$ & 0.020 \\
\hline Apathy & 0.254 & $-0.028,0.535$ & 0.076 & -0.107 & $-0.026,0.005$ & 0.169 \\
\hline Age & -0.018 & $-0.066,0.030$ & 0.465 & -0.079 & $-0.110,-0.049$ & $<0.001$ \\
\hline EYO & -0.059 & $-0.111,-0.008$ & 0.024 & -0.072 & $-0.104,-0.039$ & $<0.001$ \\
\hline Apathy X EYO & -0.308 & $-0.631,0.015$ & 0.061 & -0.027 & $-0.044,-0.010$ & 0.002 \\
\hline Appetite & -0.006 & $-0.025,0.012$ & 0.497 & -0.005 & $-0.024,0.013$ & 0.575 \\
\hline Age & -0.056 & $-0.087,-0.025$ & 0.001 & -0.076 & $-0.107,-0.045$ & $<0.001$ \\
\hline EYO & -0.020 & $-0.050,0.010$ & 0.181 & -0.076 & $-0.109,-0.053$ & $<0.001$ \\
\hline Appetite X EYO & 0.014 & $-0.005,0.033$ & 0.142 & 0.005 & $-0.011,0.021$ & 0.526 \\
\hline Delusion & -0.136 & $-0.325,0.053$ & 0.154 & -0.080 & $-0.422,0.263$ & 0.646 \\
\hline Age & -0.056 & $-0.086,-0.025$ & 0.001 & -0.082 & $-0.112,-0.051$ & $<0.001$ \\
\hline EYO & -0.017 & $-0.046,0.012$ & 0.254 & -0.067 & $-0.099,-0.034$ & $<0.001$ \\
\hline Delusion X EYO & -0.022 & $-0.051,0.008$ & 0.147 & 0.003 & $-0.029,0.035$ & 0.844 \\
\hline Depression & 0.014 & $-0.011,0.040$ & 0.281 & -0.213 & $-0.038,-0.004$ & 0.015 \\
\hline Age & -0.053 & $-0.084,-0.022$ & 0.001 & -0.080 & $-0.110,-0.050$ & $<0.001$ \\
\hline EYO & -0.026 & $-0.057,0.005$ & 0.093 & -0.078 & $-0.090,-0.045$ & 0.001 \\
\hline Depression X EYO & 0.012 & $-0.033,0.009$ & 0.270 & -0.042 & $-0.064,-0.019$ & $<0.001$ \\
\hline Disinhibition & 0.237 & $-0.007,0.480$ & 0.056 & 0.345 & $-0.016,0.085$ & 0.180 \\
\hline Age & -0.032 & $-0.069,0.003$ & 0.092 & -0.080 & $-0.111,-0.050$ & $<0.001$ \\
\hline EYO & -0.049 & $-0.090,-0.007$ & 0.022 & -0.087 & $-0.126,-0.048$ & $<0.001$ \\
\hline Disinhibition X EYO & -0.324 & $-0.642,0.006$ & 0.078 & -0.148 & $-0.372,0.007$ & 0.196 \\
\hline Irritability & 0.001 & $-0.022,0.024$ & 0.948 & 0.004 & $-0.013,0.021$ & 0.619 \\
\hline Age & -0.048 & $-0.080,-0.017$ & 0.003 & -0.081 & $-0.111,-0.051$ & $<0.001$ \\
\hline EYO & -0.023 & $-0.022,0.007$ & 0.133 & -0.077 & $-0.016,-0.044$ & $<0.001$ \\
\hline Irritability X EYO & -0.023 & -0.045 .0 .001 & 0.078 & -0.028 & $-0.048,-0.008$ & 0.006 \\
\hline Motor & -0.518 & $-1.47,0.535$ & 0.332 & -0.138 & $-0.313,0.004$ & 0.123 \\
\hline Age & -0.579 & $-2.37,1.22$ & 0.324 & -0.078 & $-0.109,-0.047$ & $<0.001$ \\
\hline EYO & -0.109 & $-0.190,-0.011$ & 0.028 & -0.071 & $-0.104,-0.037$ & $<0.001$ \\
\hline Motor X EYO & -0.370 & $-0.818,0.079$ & 0.106 & -0.018 & $-0.048,0.013$ & 0.263 \\
\hline Sleep changes & -0.001 & $-0.032,0.029$ & 0.928 & -0.008 & $-0.024,0.007$ & 0.279 \\
\hline
\end{tabular}


Table 3 Associations of interactions of NPI-Q and EYO with global $\left[{ }^{18} \mathrm{~F}\right] \mathrm{FDG}$ uptake in cognitively intact DIAD mutation carriers and non-carriers (Continued)

\begin{tabular}{llllllll}
\hline & \multicolumn{2}{l}{ DIAD mutation non-carriers } & & \multicolumn{2}{l}{ DIAD mutation carriers } \\
\cline { 2 - 3 } & Regression estimate & $\mathbf{9 5 \%} \mathrm{Cl}$ & $\boldsymbol{p}$ value & & Regression estimate & $\mathbf{9 5 \%} \mathrm{Cl}$ & $\boldsymbol{p}$ value \\
\hline Age & -0.054 & $-0.086,-0.023$ & 0.001 & & -0.075 & $-106,-0.045$ & $<0.001$ \\
EYO & -0.019 & $-0.049,0.011$ & 0.203 & & -0.074 & $-0.107,-0.041$ & $<0.001$ \\
Sleep changes X EYO & -0.015 & $-0.091,0.017$ & 0.317 & -0.009 & $-0.011,0.013$ & 0.424 \\
\hline
\end{tabular}

Mixed Model with family-level random-effects, corrected for 2-way interactions (NPI-Q sub-scales $\times$ Age \& Age $\times$ EYO), 3-way interaction (NPI-Q sub-scales $\times$ Age $\times \mathrm{EYO}$ ), gender, $A P O E$, education and family mutation type

The default mode network (DMN), which comprises the PCC, vmPFC and inferior parietal lobes, plays a vital role in episodic memory processing and decreased metabolism in the DMN is observed early in AD [34-36]. The salience network (SN), which is critical in detecting and integrating behavioural and emotional stimuli, has key nodes in the insular cortex and modulates the switch between the DMN and the central executive network [37, 38]. Impairment of the $\mathrm{SN}$ can lead to numerous neuropsychiatric disorders such as psychosis [39] and depression [40]. Brain metabolic dysfunctions within the SN are also related to NPS in AD [41]. Therefore, our findings of the association between NPS and more rapid FDG uptake decline in the PCC, vmPFC, parietal lobes, and right insula in DIAD mutation carriers with shorter EYO of AD dementia support the link between early NPS and limbic structures and brain regions involved in early AD pathophysiology.

While there is heterogeneity in the neuropsychiatric manifestations in AD, certain NPS tend to co-express.

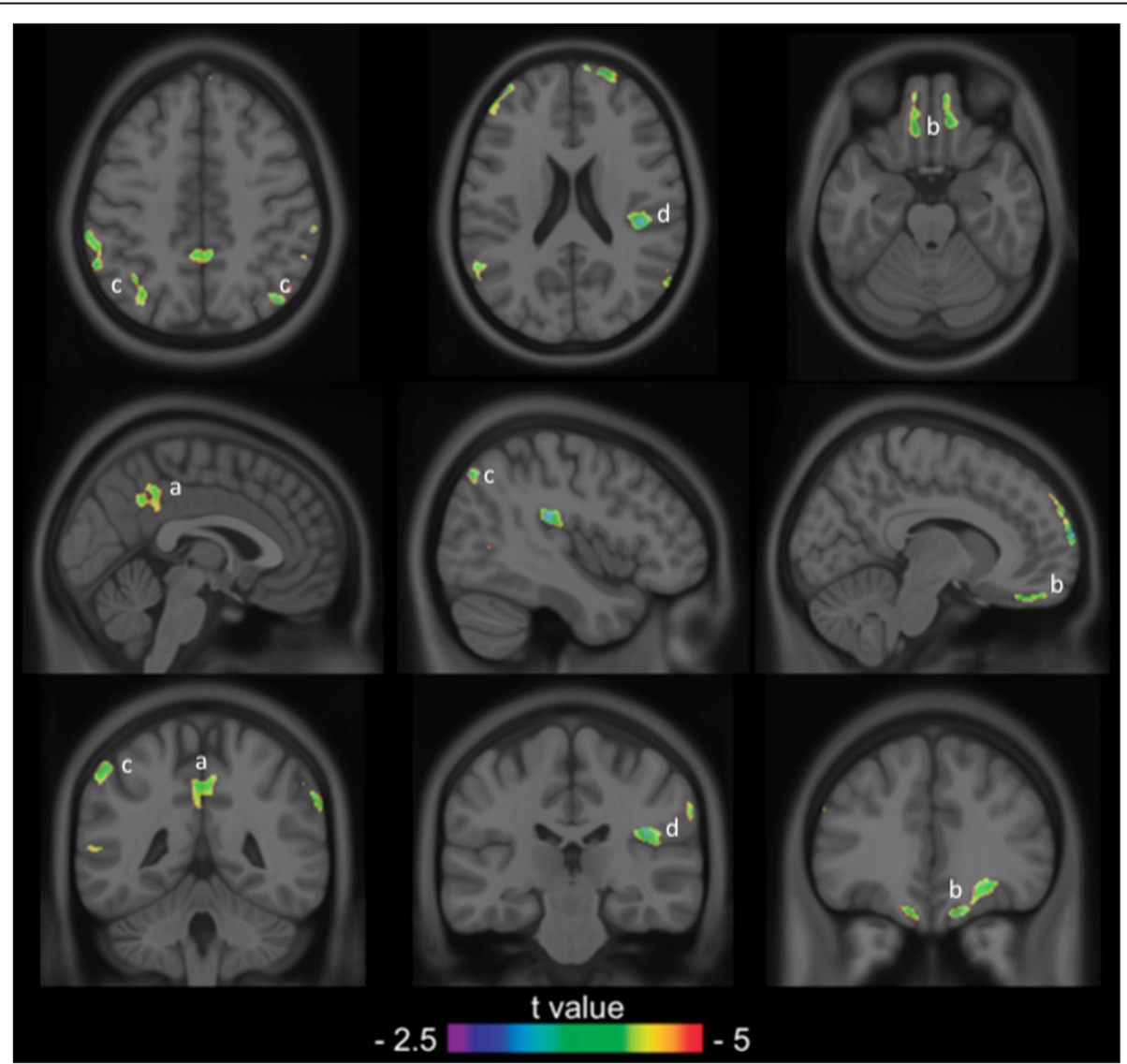

Fig. 1 Higher NPI-Q and shorter EYO of AD were associated with higher $\left[{ }^{18} \mathrm{~F}\right] \mathrm{FDG}$ uptake decline in DIAD mutation carriers. Statistical parametric map overlaid on a structural MRI scan shows regions in the PCC (a), vmPFC (b), bilateral parietal lobes (c) and right Al (d) where higher $\left[{ }^{18} \mathrm{~F}\right] \mathrm{FDG}$ uptake decline was found in cognitively normal DIAD mutation carriers with higher NPI-Q scores and shorter EYO of AD. The analysis was corrected for gender, education, APOE \&4 status and family mutation type (APP, PSEN1 and PSEN2) and multiple comparisons were corrected using random field theory at $p<0.001$. AD: Alzheimer disease; Al: anterior insula; DIAD: dominantly inherited Alzheimer's disease; EYO: estimated years to onset; $\left[{ }^{18} \mathrm{~F}\right] \mathrm{FDG}$ : $\left[{ }^{18}\right.$ F]fluorodeoxyglucose; NPI-Q: Neuropsychiatric Inventory; PCC: posterior cingulate cortex; vmPFC: ventromedial prefrontal cortex 
Table 4 Factors of neuropsychiatric subsyndromes of the cognitively intact DIAD mutation carriers

\begin{tabular}{llccc}
\hline & \multicolumn{3}{l}{ Neuropsychiatric Subsyndromes } \\
\cline { 2 - 4 } & $\mathbf{1}$ & $\mathbf{2}$ & $\mathbf{3}$ & $\mathbf{4}$ \\
\hline Agitation & 0.867 & & & 0.421 \\
Anxiety & & 0.477 & & \\
Apathy & & 0.785 & & \\
Appetite & & & & \\
Delusion & & & 0.908 & \\
Depression & 0.456 & 0.577 & & \\
Disinhibition & 0.822 & & & \\
Irritability & 0.692 & & 0.410 & \\
Motor & & 0.797 & & \\
Sleep Changes & & 0.489 & 0.564 & \\
\hline
\end{tabular}

Factor analysis identified the above 4 components which explained $70 \%$ of the variance

Hence, several neuropsychiatric subsyndromes have been identified to characterise the clustering of NPS [42] in AD. In our study, the exploratory factor analysis revealed four neuropsychiatric subsyndromes (Table 4) and among them, only the neuropsychiatric subsyndrome "agitation, disinhibition, irritability and depression" was associated with metabolic decline in cognitively intact DIAD mutation carriers with shorter EYO of AD dementia. This is consistent with findings from a systemic review of behavioural and psychological subsyndromes among elderly individuals with sporadic $\mathrm{AD}$, where 34 different clusters were found and agitation/aggression, depression, anxiety and irritability were most commonly clustered together [43]. In addition, delusion and hallucinations, depression and anxiety, agitation and irritability, and euphoria and disinhibition tend to be frequently associated symptoms. Hence, our finding is consistent with the current evidence of neuropsychiatric subsyndromes in $\mathrm{AD}$. In addition, given that the currently reported subsyndromes are mostly defined among elderly individuals with sporadic AD, our results further advanced the field by identifying specific neuropsychiatric subsyndromes among younger cognitively intact DIAD mutation carriers who are destined to develop AD. However, this will need to be confirmed in future studies in a different cohort using different methodological approaches.

The neurobiology of agitation, disinhibition, irritability and depression is closely linked to dysfunctions within the PCC, vmPFC, bilateral parietal lobes and right insular. The vmPFC regulates behavioural responses such as changing reinforcement contingencies and emotional processing and regulation [44, 45]. Irritability is linked to abnormal emotional processing associated with vmPFC and PCC, while behavioral disinhibition and prominent emotional lability are linked to lesions in the vmPFC. Dysfunctions within the vmPFC and PCC, which are part of the neural network involved in the modulation of normal emotional behaviour, also lead to affective disorders and depressive symptoms [46]. The insular plays a key role in producing appropriate behavioral responses in a person by integrating affective, homeostatic, and higher-order cognitive processes [47]. Irritability is associated with dysfunctions within the

Table 5 Neuropsychiatric symptoms (Factors) among cognitively intact DIAD mutation carriers

\begin{tabular}{|c|c|c|c|}
\hline & Regression estimate & $95 \% \mathrm{Cl}$ & $p$ value \\
\hline Age & -0.076 & $-0.016,-0.002$ & $<0.001$ \\
\hline EYO & -0.070 & $-0.101,-0.038$ & $<0.001$ \\
\hline \multirow[t]{2}{*}{ Factor 1} & \multicolumn{3}{|c|}{ Agitation + Disinhibition + Irritability + Depression } \\
\hline & 0.010 & $-0.024,0.022$ & 0.367 \\
\hline \multirow[t]{2}{*}{ Factor 2} & \multicolumn{3}{|c|}{ Anxiety + Apathy + Depression + Motor + Sleep changes } \\
\hline & -0.026 & $-0.049,-0.004$ & 0.023 \\
\hline \multirow[t]{2}{*}{ Factor 3} & \multicolumn{3}{|c|}{ Delusion + Sleep changes + Irritability } \\
\hline & 0.008 & $-0.014,0.031$ & 0.478 \\
\hline \multirow[t]{2}{*}{ Factor 4} & \multicolumn{3}{|l|}{ Appetite + Anxiety } \\
\hline & -0.004 & $-0.020,0.012$ & 0.481 \\
\hline \multicolumn{4}{|c|}{ 2-Way Interactions } \\
\hline Factor 1 X EYO & -0.044 & $-0.071,0.017$ & 0.001 \\
\hline Factor 2 X EYO & -0.020 & $-0.047,0.007$ & 0.140 \\
\hline Factor 3 X EYO & 0.006 & $-0.020,0.033$ & 0.637 \\
\hline Factor 4 X EYO & -0.005 & $-0.026,0.016$ & 0.649 \\
\hline
\end{tabular}

Mixed Model with family-level random-effects, corrected for 2-way interactions of Factors (1 to 4) X age, 3-way interactions Factors (1 to 4$) \mathrm{X} \mathrm{Age} \mathrm{X} \mathrm{EYO,} \mathrm{gender,}$ $A P O E$, education and family mutation type 
insular [48] while AD patients with agitation also appear to have dysfunctions within the frontal cortex, anterior cingulate cortex, orbitofrontal cortex, amygdala, and insula [49]. While we found that the neuropsychiatric subsyndrome of agitation, disinhibition, irritability and depression is linked to regional metabolic decline in cognitively normal DIAD mutation carriers, further studies are needed to evaluate if this subsyndrome also identifies cognitively normal individuals with an increased risk of pathological progression in sporadic AD.

The main strength of the present longitudinal study was the inclusion of preclinical DIAD mutation carriers who had AD pathology and were destined to develop $\mathrm{AD}$ in future. This allows the study of associations between NPS, metabolism and effects of increasing AD pathology over time (EYO). Furthermore, given that individuals may be susceptible to NPS presentations due to genetic, family and environmental factors, or being at risk for DIAD, employing both DIAD mutation carriers and non-carriers enables the control of these factors.

There were limitations in our study. First, while NPI$\mathrm{Q}$ is commonly used to detect NPS in AD patients, the NPI-Q is not developed for patients with prodromal or preclinical AD. Certain items of the NPI-Q may also be more relevant than others in a young cognitively intact cohort. Hence, the sensitivity of NPI-Q in identifying early NPS in cognitively intact individuals remains unclear. In addition, given that the NPI-Q is based on responses from an informed caregiver, the NPI-Q scores may not accurately reflect the NPS of study participants. In this regard, the mild behavioral impairment checklist (MBI-C) $[50,51]$ is a 34-item instrument that is sensitive in detecting $\mathrm{MBI}$ in people with $\mathrm{MCI}$, a construct that characterises the emergence of sustained NPS in predementia populations as a precursor to cognitive decline and dementia. Other scales that are potentially relevant include the Hospital Anxiety and Depression Scale (HADS) [52] and the Depression Anxiety Stress-Scale (DASS) [53]. Future studies of NPS in pre-dementia individuals should consider including these scales. Second, the NPI-Q total and sub-scale scores of our study population were relatively low, which may be due to the limitation of the NPI-Q in measuring NPS in cognitively intact individuals. While our findings may highlight the relevance of NPS with metabolism, where a small severity of NPS is associated with a big impact on metabolic decline, this needs to be confirmed in future studies. Third, while examining the associations of NPS with metabolic decline in a cohort of DIAD mutation carriers who were destined to develop AD, we did not specifically study the associations between NPS and AD biomarkers such as beta-amyloid and tau. Given the emerging evidence showing that NPS are associated with $\mathrm{AD}$ pathophysiology in preclinical and MCI individuals
[4, 54], future longitudinal studies are needed to determine this relationship. Last, there may be potential hazards of interpreting statistical constructs as theoretical constructs in the factor analytic literature [55]. Furthermore, a systemic review has found a relatively low concordance of the composition of NPI clusters among available evidence although some consistent associations of specific symptoms defining potential subsyndromes in AD across studies had been observed [56]. Acknowledging this limitation, a novel version of principal component analysis that mitigates excessive floor effects in NPI scores has been developed for more robust identification of neurobehavioral subsyndromes [57]. Therefore, future studies could use this approach to test the replicability of the associations between neurobehavioral subsyndromes and metabolic decline reported in this study.

\section{Conclusion}

Our findings support the emerging conceptual framework that NPS may be early clinical presentations of AD pathophysiology progression. Given that early NPS may contribute to the characterization of the preclinical $\mathrm{AD}$ stage, cognitively intact individuals presenting with NPS can be identified earlier so as to allow a personalized and timely preventive intervention.

\section{Abbreviations}

AD: Alzheimer's disease; CDR: Clinical dementia rating; CSF: Cerebrospinal fluid; DIAD: Dominantly inherited AD; DIAN: Dominantly Inherited Alzheimer's Network; EYO: Estimated years to symptom onset;

FDG: $\left[{ }^{18} \mathrm{~F}\right]$ Flurodeoxyglucose; MBI: Mild behavioral impairment; MBI-C: Mild behavioral impairment checklist; $\mathrm{MCl}$ : Mild cognitive impairment; NACC: National Alzheimer's Coordinating Center; NPI-Q: Neuropsychiatric inventory-questionnaire; NPS: Neuropsychiatric symptoms; PCC: Posterior cingulate cortex; PET: Positron emission tomography; SUVR: Standardized uptake value ratio; vmPFC: Ventral medial prefrontal cortex

\section{Acknowledgements}

Data collection and sharing for this project was supported by The Dominantly Inherited Alzheimer's Network (DIAN, U19AG032438) funded by the National Institute on Aging (NIA), the German Center for Neurodegenerative Diseases (DZNE), Raul Carrea Institute for Neurological Research (FLENI), Partial support by the Research and Development Grants for Dementia from Japan Agency for Medical Research and Development, AMED, and the Korea Health Technology R\&D Project through the Korea Health Industry Development Institute (KHIDI). This manuscript has been reviewed by DIAN Study investigators for scientific content and consistency of data interpretation with previous DIAN Study publications. We acknowledge the altruism of the participants and their families and contributions of the DIAN research and support staff at each of the participating sites for their contributions to this study.

Kok Pin Ng was supported by the National Medical Research Council, Research Training Fellowship, Singapore.

\section{Authors' contributions}

Kok Pin Ng: Study concept, design, analysis and interpretation of data, figure illustration and manuscript drafting. Tharick A. Pascoal: Analysis and interpretation of data and manuscript draft. Sulantha Mathotaarachchi: Image data processing, analysis and interpretation of data and manuscript draft. Yiong Huak Chan: Analysis and interpretation of data and manuscript draft. Lai Jiang: Analysis and interpretation of data and manuscript draft. Joseph Therriault: Image data processing, manuscript draft. Andrea L. Benedet: Image data processing, manuscript draft. Monica Shin: Image data processing, manuscript draft. Nagaendran Kandiah: Analysis and 
interpretation of data and manuscript draft. Celia M.T. Greenwood: Analysis and interpretation of data and manuscript draft. Pedro Rosa-Neto: Study concept, design, study supervision, and critical review of manuscript for intellectual content. Serge Gauthier: Study concept, design, study supervision, and critical review of manuscript for intellectual content. The authors have read and approved the final manuscript.

\section{Funding}

Not Applicable.

\section{Availability of data and materials}

The datasets used and/or analysed during the current study are available from the Dominantly Inherited Alzheimer Network on reasonable request.

\section{Ethics approval and consent to participate}

The DIAN study was approved by the Institutional Review boards of all of the participating institutions.

\section{Consent for publication}

Informed written consent was obtained from all participants at each site.

\section{Competing interests}

Not Applicable.

\section{Author details}

'Alzheimer's Disease Research Unit, McGill Centre for Studies in Aging, McGill University, Montréal, Québec, Canada. ${ }^{2}$ Translational Neuroimaging Laboratory, The McGill University Research Centre for Studies in Aging, Montreal, Canada. ${ }^{3}$ Department of Neurology, National Neuroscience Institute, Singapore City, Singapore. ${ }^{4}$ Biostatistics Unit, Yong Loo Lin School of Medicine, National University of Singapore, Singapore City, Singapore. ${ }^{5}$ Lady Davis Institute, McGill University, Montreal, Canada. ${ }^{6}$ Department of Epidemiology, Biostatistics and Occupational Health, McGill University, Montreal, Canada.

\section{Received: 3 August 2020 Accepted: 1 December 2020}

\section{Published online: 04 January 2021}

\section{References}

1. Lyketsos CG, Carrillo MC, Ryan JM, Khachaturian AS, Trzepacz P, Amatniek J, et al. Neuropsychiatric symptoms in Alzheimer's disease. Alzheimers Dement. 2012;7:532-9.

2. Lyketsos CG, Lopez O, Jones B, Fitzpatrick AL, Breitner J, DeKosky S. Prevalence of neuropsychiatric symptoms in dementia and mild cognitive impairment: results from the cardiovascular health study. JAMA. 2002;288: $1475-83$.

3. Vik-Mo AO, Giil LM, Ballard C, Aarsland D. Course of neuropsychiatric symptoms in dementia: 5-year longitudinal study. Int I Geriatr Psychiatry. 2018:33(10):1361-9.

4. Scaricamazza E, Colonna I, Sancesario GM, Assogna F, Orfei MD, Franchini F, et al. Neuropsychiatric symptoms differently affect mild cognitive impairment and Alzheimer's disease patients: a retrospective observational study. Neurol Sci. 2019;40(7):1377-82.

5. Fischer CE, Ismail Z, Schweizer TA. Delusions increase functional impairment in Alzheimer's disease. Dement Geriatr Cogn Disord. 2012;33:393-9.

6. Teng E, Lu PH, Cummings JL. Neuropsychiatric symptoms are associated with progression from mild cognitive impairment to Alzheimer's disease. Dement Geriatr Cogn Disord. 2007:24:253-9.

7. Karttunen K, Karppi P, Hiltunen A, Vanhanen M, Välimäki T, Martikainen J, et al. Neuropsychiatric symptoms and quality of life in patients with very mild and mild Alzheimer's disease. Int I Geriatr Psychiatry. 2011;26:473-82.

8. Geda YE, Roberts RO, Mielke MM, Knopman DS, Christianson TJH, Pankratz VS, et al. Baseline neuropsychiatric symptoms and the risk of incident mild cognitive impairment: a population-based study. Am J Psychiatry. 2014;171: 572-81.

9. Masters MC, Morris JC, Roe CM. Noncognitive symptoms of early Alzheimer disease: a longitudinal analysis. Neurology. 2015;84:617-22.

10. Wise EA, Rosenberg PB, Lyketsos CG, Leoutsakos JM. Time course of neuropsychiatric symptoms and cognitive diagnosis in National Alzheimer's coordinating centers volunteers. Alzheimers Dement (Amst). 2019;11:333-9.
11. Burhanullah MH, Tschanz JAT, Peters ME, Leoutsakos JM, Matyi J, Lyketsos $C G$, et al. Neuropsychiatric symptoms as risk factors for cognitive decline in clinically normal older adults: the Cache County study. Am J Geriatr Psychiatry. 2020;28(1):64-71.

12. Ismail Z, Smith EE, Geda Y, Sultzer D, Brodaty H, Smith G, et al. Neuropsychiatric symptoms as early manifestations of emergent dementia: provisional diagnostic criteria for mild behavioral impairment. Alzheimers Dement. 2016;12:195-202.

13. Ng KP, Pascoal TA, Mathotaarachchi S, Chung CO, Benedet AL, Shin M, et al. Neuropsychiatric symptoms predict hypometabolism in preclinical. Alzheimer disease. Neurology. 2017;88:1814-21.

14. Bekris $L M, Y u C E$, Bird TD, Tsuang DW. Review article: genetics of Alzheimer disease. J Geriatr Psychiatry Neurol. 2010;23(4):213-27.

15. Jack CR, Knopman DS, Jagust WJ, Petersen RC, Weiner MW, Aisen PS, et al. Tracking pathophysiological processes in Alzheimer's disease: an updated hypothetical model of dynamic biomarkers. Lancet Neurol. 2013;12:207-16.

16. Bateman RJ, Xiong C, Benzinger TLS, Fagan AM, Goate A, Fox NC, et al. Clinical and biomarker changes in dominantly inherited Alzheimer's disease. N Engl J Med. 2012:367:795-804.

17. Fagan AM, Xiong C, Jasielec MS, Bateman RJ, Goate AM, Benzinger TLS, et al. Longitudinal change in CSF biomarkers in autosomal-dominant Alzheimer's disease. Sci Transl Med. 2014;6(226):226ra30.

18. Ringman JM, Liang $\sqcup$, Zhou Y, Vangala S, Teng E, Kremen S, et al. Early behavioural changes in familial Alzheimer's disease in the dominantly inherited Alzheimer network. Brain. 2015;138:1036-45.

19. Ringman JM, Monsell S, Ng DW, Zhou Y, Nguyen A, Coppola G, et al. Neuropathology of autosomal dominant Alzheimer disease in the national Alzheimer coordinating center database. J Neuropathol Exp Neurol. 2016;75: 284-90.

20. Morris JC, Aisen PS, Bateman RJ, Benzinger TL, Cairns NJ, Fagan AM, et al. Developing an international network for Alzheimer's research: the dominantly inherited Alzheimer network. Clin Investig (Lond). 2012;2(10): 975-84

21. Morris JC. The clinical dementia rating (CDR): current version and scoring rules. Neurology. 1993;43:2412-4.

22. Folstein MF, Folstein SE, McHugh PR. "Mini-mental state". A practical method for grading the cognitive state of patients for the clinician. J Psychiatr Res. 1975;12:189-98.

23. Storandt M, Balota DA, Aschenbrenner AJ, Morris JC. Clinical and psychological characteristics of the initial cohort of the dominantly inherited Alzheimer network (DIAN). Neuropsychology. 2014;28:19-29.

24. Kaufer DI, Cummings IL, Ketchel P, Smith V, MacMillan A, Shelley T, et al. Validation of the NPI-Q, a brief clinical form of the neuropsychiatric inventory. J Neuropsychiatry Clin Neurosci. 2000;12:233-9.

25. Wang G, Coble D, McDade EM, Hassenstab J, Fagan AM, Benzinger TLS, et al. Staging biomarkers in preclinical autosomal dominant Alzheimer's disease by estimated years to symptom onset. Alzheimers Dement. 2019; 15(4):506-14.

26. Ad-Dab'bagh Y, Lyttelton O, Muehlboeck JS, Lepage C, Einarson D, Mok K, et al. The CIVET image-processing environment: a fully automated comprehensive pipeline for anatomical neuroimaging research. Proc. 12th Annu. Meet Organ Hum Brain Mapp. 2006:2266.

27. Pascoal TA, Mathotaarachchi S, Mohades S, Benedet AL, Chung C-O, Shin $\mathrm{M}$, et al. Amyloid- $\beta$ and hyperphosphorylated tau synergy drives metabolic decline in preclinical Alzheimer's disease. Mol Psychiatry. 2017:22:306-11

28. Nugent S, Croteau E, Potvin O, Castellano CA, Dieumegarde L, Cunnane SC, et al. Selection of the optimal intensity normalization region for FDG-PET studies of normal aging and Alzheimer's disease. Sci Rep. 2020;10(1):9261.

29. López Mora DA, Sampedro F, Camacho V, Fernández A, Fuentes F, Duch J, et al. Selection of reference regions to model neurodegeneration in Huntington disease by 18 F-FDG PET/CT using imaging and clinical parameters. Clin Nucl Med. 2019;44(1):e1-5.

30. R Developement Core Team. R: A Language and Environment for Statistical Computing. R Found Stat Comput. 2015;1:409.

31. Worsley K, Taylor JE, Tomaiuolo F, Lerch J. Unified univariate and multivariate random field theory. Neuroimage. 2004;23(Suppl 1):S189-95.

32. Banks SJ, Raman R, He F, Salmon DP, Ferris S, Aisen P, et al. The Alzheimer's disease cooperative study prevention instrument project: longitudinal outcome of behavioral measures as predictors of cognitive decline. Dement Geriatr Cogn Dis Extra. 2014;4(3):509-16. 
33. Krell-Roesch J, Ruider H, Lowe VJ, Stokin GB, Pink A, Roberts RO, et al. FDG PET and neuropsychiatric symptoms among cognitively normal elderly persons: the Mayo clinic study of aging. J Alzheimers Dis. 2016;53:1609-16.

34. Greicius MD, Supekar K, Menon V, Dougherty RF. Resting-state functional connectivity reflects structural connectivity in the default mode network. Cereb Cortex. 2009:19:72-8.

35. Liguori C, Chiaravalloti A, Sancesario G, Stefani A, Sancesario GM, Mercuri NB, et al. Cerebrospinal fluid lactate levels and brain [18F]FDG PET hypometabolism within the default mode network in Alzheimer's disease. Eur J Nucl Med Mol Imaging. 2016;43(11):2040-9.

36. Mevel K, Chételat G, Eustache F, Desgranges B. The default mode network in healthy aging and Alzheimer's disease. Int J Alzheimers Dis 2011;2011: 535816

37. He X, Qin W, Liu Y, Zhang X, Duan Y, Song J, et al. Abnormal salience network in normal aging and in amnestic mild cognitive impairment and Alzheimer's disease. Hum Brain Mapp. 2014;35:3446-64.

38. Goulden N, Khusnulina A, Davis NJ, Bracewell RM, Bokde AL, McNulty JP, et al. The salience network is responsible for switching between the default mode network and the central executive network: replication from DCM. Neuroimage. 2014;99:180-90.

39. Palaniyappan L, Liddle PF. Does the salience network play a cardinal role in psychosis? An emerging hypothesis of insular dysfunction. J Psychiatry Neurosci. 2012;37(1):17-27.

40. Manoliu A, Meng C, Brandl F, Doll A, Tahmasian M, Scherr M, et al. Insular dysfunction within the salience network is associated with severity of symptoms and aberrant inter-network connectivity in major depressive disorder. Front Hum Neurosci. 2014;21(7):930.

41. Ballarini $T$, laccarino L, Magnani G, Ayakta N, Miller BL, Jagust WJ, et al. Neuropsychiatric subsyndromes and brain metabolic network dysfunctions in early onset Alzheimer's disease. Hum Brain Mapp. 2016;37(12):4234-47.

42. Aalten P, Verhey FRJ, Boziki M, Brugnolo A, Bullock R, Byrne EJ, et al. Consistency of neuropsychiatric syndromes across dementias: results from the European Alzheimer disease consortium - part II. Dement Geriatr Cogn Disord. 2007;25:1-8.

43. Nowrangi MA, Lyketsos CG, Rosenberg PB. Principles and management of neuropsychiatric symptoms in Alzheimer's dementia. Alzheimers Res Ther. 2015;7(1):12.

44. Bechara A, Damasio H, Damasio AR, Lee GP. Different contributions of the human amygdala and ventromedial prefrontal cortex to decision-making. J Neurosci. 1999;19:5473-81.

45. Zald DH, Andreotti C. Neuropsychological assessment of the orbital and ventromedial prefrontal cortex. Neuropsychologia. 2010;48(12):3377-91.

46. Drevets WC, Price JL, Furey ML. Brain structural and functional abnormalities in mood disorders: implications for neurocircuitry models of depression. Brain Struct Funct. 2008;213(1-2):93-118.

47. Menon V, Uddin LQ. Saliency, switching, attention and control: a network model of insula function. Brain Struct Funct. 2010;214(5-6):655-67.

48. Balthazar MLF, Pereira FRS, Lopes TM, da Silva EL, Coan AC, Campos BM, et al. Neuropsychiatric symptoms in Alzheimer's disease are related to functional connectivity alterations in the salience network. Hum Brain Mapp. 2014;35(4):1237-46.

49. Rosenberg PB, Nowrangi MA, Lyketsos CG. Neuropsychiatric symptoms in Alzheimer's disease: What might be associated brain circuits? Mol Aspects Med. 2015:43-44:25-37.

50. Ismail Z, Agüera-Ortiz L, Brodaty H, Cieslak A, Cummings J, Fischer CE, et al. The mild behavioral impairment checklist (MBI-C): a rating scale for neuropsychiatric symptoms in pre-dementia populations. J Alzheimers Dis. 2017:56:929-38

51. Mallo SC, Ismail Z, Pereiro AX, Facal D, Lojo-Seoane C, Campos-Magdaleno $M$, et al. Assessing mild behavioral impairment with the mild behavioral impairment-checklist in people with mild cognitive impairment. J Alzheimers Dis. 2018;66:83-95.

52. Zigmond AS, Snaith RP. The hospital anxiety and depression scale. Acta Psychiatr Scand. 1983;67(6):361-70.

53. Lovibond SH, Lovibond PF. Manual for the depression anxiety stress scales. Behav Res Ther. 1995;33(3):335-43.

54. Lussier FZ, Pascoal TA, Chamoun M, Therriault J, Tissot C, Savard M, et al. Mild behavioral impairment is associated with $\beta$-amyloid but not tau or neurodegeneration in cognitively intact elderly individuals. Alzheimers Dement. 2020;16:192-9.
55. Fried El. Lack of theory building and testing impedes progress in the factor and network literature. 2020. Available from: www.eiko-fried.com. DOI https://doi.org/10.17605/OSF.IO/6CTS9.

56. Canevelli M, Adali N, Voisin T, Soto ME, Bruno G, Cesari M, et al. Behavioral and psychological subsyndromes in Alzheimer's disease using the neuropsychiatric inventory. Int J Geriatr Psychiatry. 2013;28(8):795-803.

57. Hellton KH, Cummings J, Vik-Mo AO, Nordrehaug JE, Aarsland D, Selbaek G, et al. The truth behind the Zeros: a new approach to principal component analysis of the neuropsychiatric inventory. Multivariate Behav Res. 2020:1-16.

\section{Ready to submit your research? Choose BMC and benefit from:}

- fast, convenient online submission

- thorough peer review by experienced researchers in your field

- rapid publication on acceptance

- support for research data, including large and complex data types

- gold Open Access which fosters wider collaboration and increased citations

- maximum visibility for your research: over $100 \mathrm{M}$ website views per year

At BMC, research is always in progress.

Learn more biomedcentral.com/submissions 